\title{
Comunidades de quitones (Mollusca: Polyplacophora) de la Bahía de La Paz, Baja California Sur, México
}

\author{
Cedar I. García Ríos ${ }^{1}$ \& Migdalia Álvarez Ruiz ${ }^{2}$ \\ 1 Departamento de Biología, Universidad de Puerto Rico en Humacao, Humacao, Puerto Rico 00792; cgarcia@www. \\ uprh.edu \\ 2 Departamento de Biología, Universidad de Puerto Rico en Ponce, Ponce, Puerto Rico 00732; biomar@coqui.net
}

\author{
Recibido 16-VII-2002. Corregido 16-IX-2005. Aceptado 19-X-2006.
}

\begin{abstract}
Polyplacophoran communities (Mollusca: Polyplacophora) at Bahía de La Paz, Baja California Sur, México. Eight species of polyplacophorans have been reported from La Bahía de la Paz, Baja California Sur, Mexico. We add Lepidochitona beanii, Chaetopleura lurida, Stenoplax limaciformis, S. mariposa, Lepidozona clathrata, L. serrata and Acanthochitona arragonites, increasing the known number of species to 15. Ordination analysis of five chiton communities at the site suggests a correlation of wave exposure to species composition and diversity: communities with intermediate wave exposure have more species (richness) and higher diversity (Shannon's index). Rev. Biol. Trop. 55 (1): 177-182. Epub 2007 March. 31.
\end{abstract}

Key words: Mollusca, Polyplacophora, chiton, Gulf of California, Bahía de La Paz, México.

Los quitones son moluscos abundantes en la franja mesolitoral y la zona sublitoral alta con fondos rocosos y coralinos (Hyman 1967, Kaas y Van Belle 1985, Slieker 2000). A pesar de su diversidad y abundancia son subestimados en los estudios faunísticos realizados en México (Reyes y Salcedo 2002). Esto puede deberse a varios factores, tales como la ausencia de especies con importancia comercial, los hábitos crípticos de la mayoría de las especies y las dificultades de recolectar en la zona del embate del oleaje de las costas rocosas, donde los quitones son abundantes.

La Bahía de La Paz está ubicada en la costa sureste de la Península de Baja California $\left(24.1^{\circ}-24.8^{\circ} \mathrm{N}\right.$ y $\left.110.2^{\circ}-110.8^{\circ} \mathrm{W}\right)$. En el extremo sur de la Bahía se encuentra la Ensenada de la Paz, zona poco profunda protegida del oleaje por El Mogote, una barrera de arena (Fig. 1). Al norte de la Ensenada, ubicado entre la isla Espíritu Santo y Tecolote, se encuentra el Canal de San Lorenzo. Este canal conecta el sur de la Bahía con el Golfo de California.
Existe un gradiente de exposición a la energía del oleaje en la medida en que nos trasladamos de la Ensenada (zona protegida) hasta Tecolote (zona expuesta) (Jiménez et al. 1997).

Se han realizado varios trabajos de naturaleza taxonómica con quitones en La Paz. Es la localidad tipo de tres especies (Slieker 2000): Ischnochiton tridentatus (Pilsbry 1893), Callistochiton elenensis (Sowerby 1832) y Acanthochitona exquisita (Pilsbry 1893). Otras seis especies estaban documentadas: Nuttallina crossota (Berry 1956) en Kaas y Van Belle (1985), Stenoplax circumsenta (Berry 1956) en Kaas y Van Belle (1987), A. avicula (Carpenter 1866) en Burghardt y Burghardt (1969), y Watters (1990), A. imperatrix (Watters 1981) en Watters (1990), Chiton virgulatus (Sowerby 1840) y A. exquisita (Pilsbry 1893) en Holguin y García (1997). El objetivo del presente trabajo es ampliar la lista de especies de quitones presentes en La Paz y describir la estructura de las comunidades de quitones en cinco estaciones, para interpretar sus similitudes y diferencias. 


\section{MATERIALES Y MÉTODOS}

Se realizaron cinco viajes de recolección a sendas estaciones durante los meses de diciembre de 2001 y enero de 2002. Cuatro de las estaciones están localizadas en el sur de La Bahía de La Paz: El Malecón (2409'57' N, 110¹8'49" W), Pichilingue $\left(24^{\circ} 15^{\prime} 37^{\prime \prime} \mathrm{N}, 110^{\circ} 18^{\prime} 54^{\prime \prime}\right.$ W), La Balandra $\left(24^{\circ} 19^{\prime} 16^{\prime \prime} \mathrm{N}, 110^{\circ} 19^{\prime} 33^{\prime \prime}\right.$ W) y Tecolote $\left(24^{\circ} 21^{\prime} 09^{\prime \prime}\right.$ N, $\left.110^{\circ} 17^{\prime} 49^{\prime \prime} \mathrm{W}\right)$ (Fig. 1). Como estación de mayor exposición al oleaje se seleccionó una costa de guijarros localizada en la playa Sargento $\left(24^{\circ} 03^{\prime} 37^{\prime \prime} \mathrm{N}\right.$, $110^{\circ} 59^{\prime} 15^{\prime \prime} \mathrm{W}$ ).

En todas las estaciones se recolectó un total de diez horas/ persona. Toda la recolección se realizó a profundidades iguales o menores a $1 \mathrm{~m}$ de profundidad. Todos los organismos se recolectaron despegándolos de rocas que tenían entre 3 y $75 \mathrm{~cm}$ de diámetro. Las rocas donde encontramos quitones estaban parcialmente enterradas en arena. Otros hábitats fueron examinados, pero no localizamos en ellos ningún ejemplar. Los lotes de quitones recolectados fueron relajados y aplanados para su preservación usando alcohol etílico diluido con agua de mar al $70 \%$.

La identificación de los quitones se realizó utilizando la serie de monografías taxonómicas de Kaas y Van Belle (1985, 1987, 1990 y 1994), Watters (1990) y las guías regionales de Brusca (1980) y Keen (1971). Los datos taxonómicos se presentan utilizando el esquema de clasificación de Kaas y Van Belle (1998). Se depositaron ejemplares de referencia en el Zoologische Staatssammlung München (ZSM), Alemania. Los ejemplares restantes se depositaron en la Colección Biológica de la Universidad de Puerto Rico en Humacao (CBUPRH).

Se compararon las comunidades calculando la riqueza de las especies y la diversidad (Diversidad de Shannon, H') según Brower et al. (1998). Se realizaron comparaciones gráficas utilizando la técnica de ordenación BrayCurtis. Este tratamiento permite interpretar los patrones de segregación de las comunidades

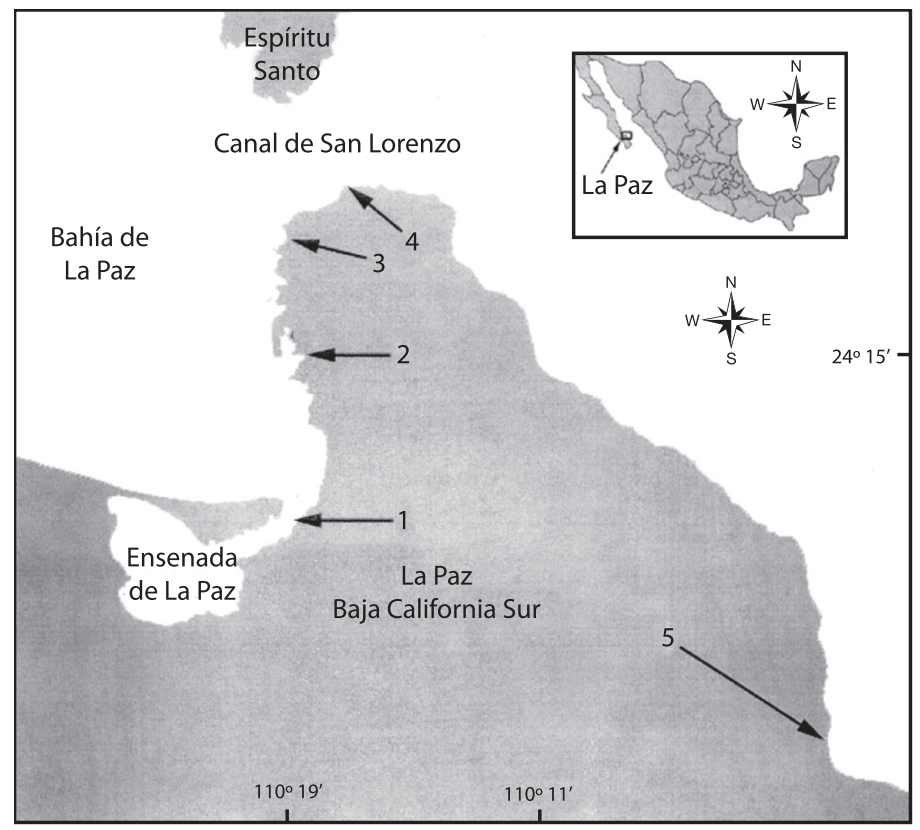

Fig. 1. Localización de las cinco estaciones de colección en La Paz, Baja California Sur, México: 1- Malecón, 2- Pichilingue, 3- Balandra, 4- Tecolote y 5-Sargento.

Fig. 1. Location of five collection stations in La Paz, Baja California Sur, México: 1- Malecón, 2- Pichilingue, 3- Balandra, 4- Tecolote and 5- Sargento. 
con información ambiental existente, según se describe en Gauch (1982). Para preparar la matriz primaria de la que se derivó la gráfica de ordenación se realizó una normalización de las especies (calculando el porcentaje de la presencia de cada especie en cada estación). La distancia entre los puntos en la gráfica de ordenación es proporcional al porcentaje de disimilaridad (distancia Sorensen) de las comunidades encontradas en cada estación. A partir de la misma matriz, con el objeto de comparar la similitud estructural de las comunidades estudiadas se preparó un dendrograma, utilizando distancia objetiva (Sorensen), según Wishart (1969).

\section{RESULTADOS}

Se recolectaron un total de 360 quitones, de los cuales se identificaron 13 especies diferentes en las estaciones localizadas en la Bahía de La Paz (Cuadro 1). De la totalidad recolectada, casi el 50\% pertenece a sólo dos especies: I. tridentatus (108 individuos) y Stenoplax mariposa (70 individuos). Éstas dos especies junto a Lepidozona serrata (51) y $C$. elenensis (29) están presentes en cuatro de las cinco estaciones. Ninguna especie se encontró presente en las cinco estaciones.

La estación donde se recuperó el mayor número de ejemplares fue la estación en playa

\section{CUADRO 1}

Frecuencia de individuos, riqueza de especies y diversidad (Índice de Shannon) de los quitones (Mollusca: Polyplacophora) de La Paz, Baja California Sur, México

TABLE 1

Number of individuals, species richness, and Shannon diversity index of chitons (Mollusca: Polyplacophora) at La Paz, Baja California Sur, México

\begin{tabular}{|c|c|c|c|c|}
\hline & & & Estación & \\
\hline Especie & Malecón & Pichilingue & Balandra & Tecolote \\
\hline
\end{tabular}

Lepidochitona beanii

2

Nuttallina crossota

11

Chaetopleura lurida

Ischnochiton tridentatus

14

Stenoplax limaciformis

Stenoplax mariposa

6

19

Lepidozona clathrata

Lepidozona serrata

Callistochiton elenensis

4

Chiton virgulatus

Acanthochitona arragonites

Acanthochitona avicula

Acanthochitona exquisita

Total de individuos

12

Riqueza de especies

Diversidad de Shannon, H'

2

6

6

2

2

1

6

23

13

37

1

1

1

1

21

5

1

18

$\begin{array}{ccc}57 & 59 & 146 \\ 5 & 7 & 7 \\ 0.53 & 0.75 & 0.73\end{array}$


Sargento, fuera de la Bahía (146 individuos). Sin embargo, la estación con mayor riqueza de especies fue la localizada en Pichilingue, donde se identificaron 10 de las 13 especies encontradas (Cuadro 1). En esa estación encontramos cuatro especies que no fueron encontradas en ninguna otra.

Siete de las especies recolectadas son informes nuevos para la Bahía de La Paz, éstas son: Lepidochitona beanii (Carpenter 1857) (CBUPRH 1894), Chaetopleura lurida (Sowerby 1832) (CBUPRH 1909 y 1916), Stenoplax limaciformis (Sowerby 1832) (CBUPRH 1911 y 1918), S. mariposa (Bartsch MS, Dall 1919) (CBUPRH 1891, 1897 y 1905), Lepidozona clathrata (Reeve 1847) (ZSM 20020810 y CBUPRH 1898), L. serrata (Carpenter 1864) (CBUPRH 1899, 1906, 1912 y 1920) y Acanthochitona arragonites (Carpenter 1857) (CBUPRH 1902). De acuerdo con Reyes y Salcedo (2002) L. clathrata es una especie endémica de la costa oeste de Baja California, éste sería el primer informe para el Golfo de California.

El análisis de ordenación polar (Fig. 2) coloca en el extremo izquierdo a las comunidades de quitones coleccionadas en las estaciones en Tecolote y Sargento, las dos estaciones de mayor exposición al oleaje. En el extremo opuesto del gráfico aparece la estación en el Malecón, protegida del oleaje por el Mogote (Fig. 1). El eje 1 de esa gráfica (Fig. 2) corresponde con el gradiente de exposición a energía física descrito en los modelos de Jiménez et al. (1997), con las estaciones en las localidades de mayor energía a la izquierda y las de menor a la derecha.

La comunidad de quitones asociada a la Balandra y el Malecón presentan los valores más bajos del índice de diversidad $\left(\mathrm{H}^{\prime}=0.42 \mathrm{y}\right.$ 0.42) (Cuadro 1). Estas comunidades están asociadas a las estaciones con menor exposición a energía física. Las comunidades encontradas en Sargento y Tecolote, las de mayor energía, presentan valores intermedios de diversidad $\left(\mathrm{H}^{\prime}=\right.$ 0.66 y 0.64$)$. Corresponde a la estación de exposición intermedia, en Pichilingue, la presencia de la comunidad de quitones de mayor riqueza (10 especies) y mayor diversidad $\left(\mathrm{H}^{\prime}=0.84\right)$.

El dendrograma (Fig. 3) que compara a las cinco comunidades agrupa, por su mayor similitud, a las comunidades en Balandra y Malecón (Fig. 3). Estas comunidades comparten las tres especies presentes en el Malecón y en ambas, la especie dominante es $S$. mariposa. Ambas son las estaciones de menor riqueza de especies (Cuadro 1). El segundo par de mayor similitud es Tecolote y Sargento, las dos tienen la misma especie dominante: I. tridentatus. De las siete especies diferentes que tienen las dos estaciones, cinco son compartidas. La estación de Pichilingue tiene mayor similitud con el par Balandra y Malecón.

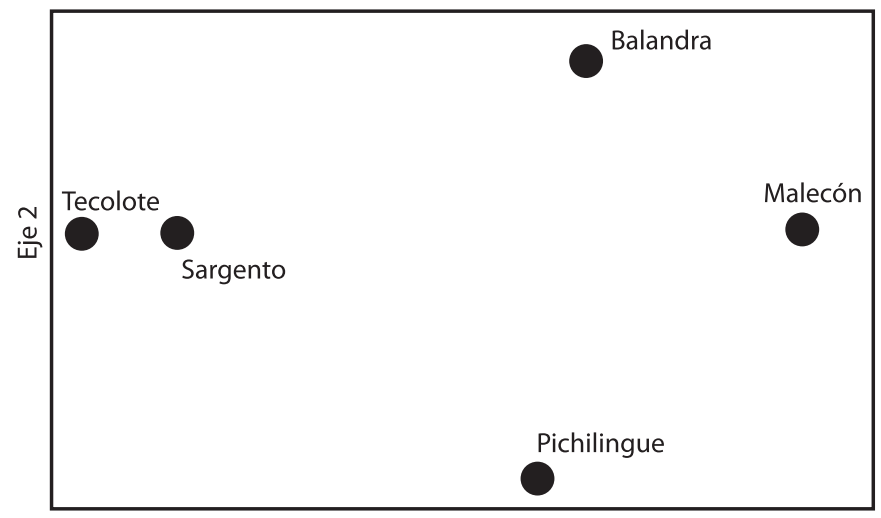

Eje 1

Fig. 2. Ordenación de cinco comunidades de quitones (Mollusca: Polyplacophora) en La Paz, Baja California Sur, México. Fig. 2. Ordination of five chiton (Mollusca: Polyplacophora) communities at La Paz, Baja California Sur, México. 


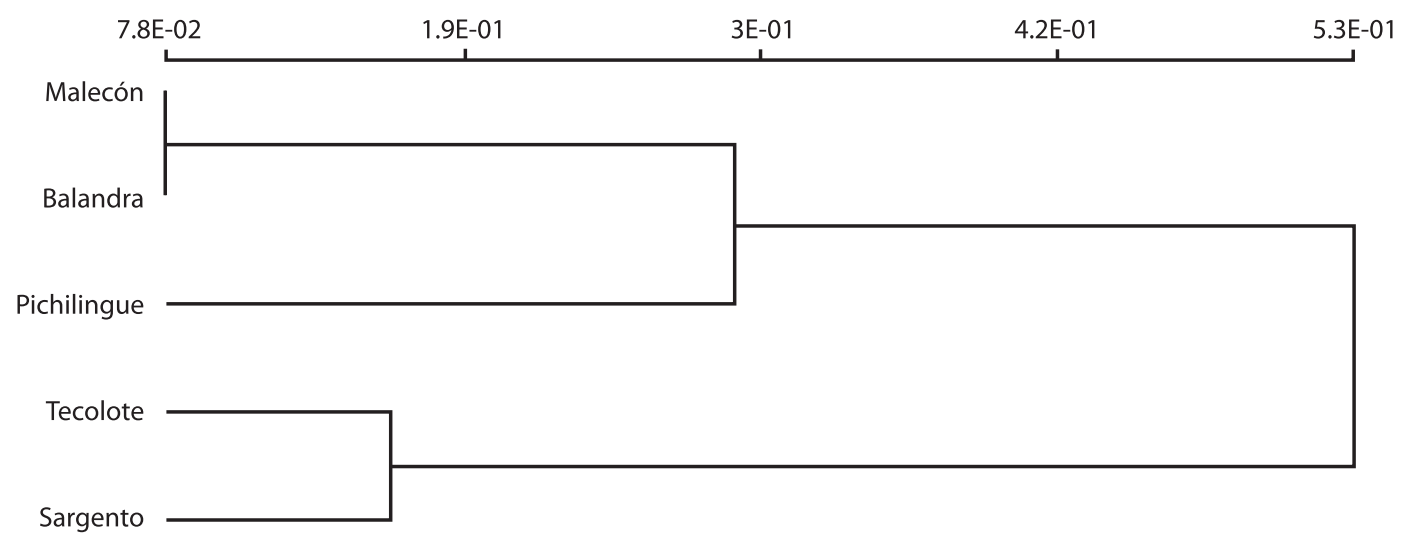

Fig. 3. Dendrograma de distancia (Sorensen) entre comunidades de quitones (Mollusca: Polyplacophora) asociadas a cinco estaciones en La Paz, Baja California Sur, México.

Fig. 3. Dendrogram (Sorensen distance) of five chiton (Mollusca: Polyplacophora) communities at La Paz, Baja California Sur, Mexico.

\section{DISCUSIÓN}

La estructura de las comunidades de quitones es diferente en cada una de las estaciones estudiadas en La Paz. Tanto la ordenación de las estaciones (Fig. 2), como los agrupamientos representadas en el dendrograma (Fig. 3) coinciden, y pueden interpretarse relacionándolos al modelo de exposición a energía física publicado por Jiménez et al. (1997). Las comunidades en los litorales rocosos tradicionalmente se ordenan por gradientes de exposición a oleaje. Corrientes litorales y olas también influencian otros factores ambientales como la sedimentación y granulometría (Menge y Branch 2001). Los quitones, en general, son sensibles a la acumulación de sedimentos que se encuentra en ambientes de poca energía, pues estos pueden congestionar sus branquias e interferir con su alimentación (Hyman 1967). Por otra parte, un oleaje extremadamente fuerte, además del efecto mecánico que ejerce sobre los organismos que impacta, mueve sobre el fondo sedimentos gruesos que tienen un efecto abrasivo sobre los moluscos y sus recursos alimentarios.

Las especies que encontramos con mayor frecuencia relativa en las comunidades de menor exposición son $S$. mariposa y $C$. elenensis. En el otro extremo, en los ambientes de mucha energía física, tanto $C$. lurida como
C. virgulatus son especies características. La estación de Pichilingue, con una exposición intermedia, tiene presentes las especies de ambos extremos y otras que no son abundantes en otras estaciones. Se propone que las condiciones intermedias de energía son las que explican la mayor riqueza y diversidad de especies en Pichilingue, y que niveles bajos o altos de energía impiden o reducen la presencia de algunas especies y reducen la diversidad de quitones. Ésta hipótesis permite explicar también la total ausencia de ejemplares en las búsquedas realizadas en ambientes con menos energía (suroeste del Malecón) y ambientes de mayor oleaje (La Ventana).

\section{AGRADECIMIENTOS}

Agradecemos a Emilio González, Olga Dorantes Salas y Luis Alberto González su ayuda en la selección y localización de las estaciones de colección. Agradecemos el apoyo logístico y los datos de campo que generosamente ofrecieron Edwin Omar Rodríguez, Gerardo Aceves y Cristina Bond (CICIMAR). Queremos reconocer la pronta respuesta y eficiente servicio para el depósito de especímenes testigo que ofreció Enrico Schwabe (ZSM). 


\section{RESUMEN}

A las ocho especies de poliplacóforos registradas en Bahía de la Paz, Baja California Sur, México, o cerca de ella, agregamos a Lepidochitona beanii, Chaetopleura lurida, Stenoplax limaciformis, S. mariposa, Lepidozona clathrata, L. serrata y Acanthochitona arragonites. El análisis de las comunidades estudiadas usando técnicas de ordenación sugiere que podría relacionarse la exposición al oleaje con la riqueza y la diversidad de especies. La comunidad recolectada en condiciones intermedias de exposición al oleaje presentó mayor número de especies y mayor diversidad de acuerdo con el índice de Shannon.

Palabras claves: Mollusca, Polyplacophora, quitón, Golfo de California, Bahía de La Paz, México.

\section{REFERENCIAS}

Brusca, R.C. 1980. Common intertidal invertebrates of the Gulf of California. University of Arizona, Phoenix, Arizona, EUA. 513 p.

Brower, J.E., J.H. Zar \& C.N. von Ende. 1998. Field and laboratory methods for general ecology. McGrawHill, Boston, Massachussetts, EUA. 273 p.

Burghardt, G.E. \& L.E. Burghardt. 1969. A collector's guide to west coast chitons. Special Publication No. 4, San Francisco Aquarium Society, San Francisco, California, EUA. 45 p.

Gauch, H.G. 1982. Multivariate analysis in community ecology. Cambridge University, Nueva York, Nueva York, EUA. 298 p.

Holguin-Quiñones, O.E. \& F.A. García-Domínguez. 1997. Lista anotada de las especies de moluscos recolectadas en la Bahía de La Paz, B.C.S., p. 93-117. In J. Urbán Ramírez \& M. Ramírez Rodríguez (eds.). La Bahía de La Paz, investigación y conservación. Universidad Autónoma de Baja California Sur, Centro Interdisciplinario de Ciencias Marinas y Scripps Institution of Oceanography. La Paz, Baja California Sur, México.

Hyman, L.H. 1967. The Invertebrates: Mollusca I (Vol. VI). McGraw-Hill, Nueva York, Nueva York, EUA. $792 \mathrm{p}$.

Jiménez-Illesca, R.A., M. Obeso-Nieblas \& D.A. Salas de León. 1997. Oceanografía física de la Bahía de la Paz, B.C.S., p. 31-41. In J. Urbán Ramírez \& M. Ramírez Rodríguez (eds.). La Bahía de La Paz, investigación y conservación. Universidad Autónoma de Baja California Sur, Centro Interdisciplinario de Ciencias Marinas y Scripps Institution of Oceanography. La Paz, Baja California Sur, México.

Kaas, P. \& R.A. Van Belle. 1985. Monograph of living chitons. Vol. 2, Suborder Ischnochitonina, Ischnochitonidae: Schizoplacinae, Callochitoninae \& Lepidochitoninae. E.J. Brill / W. Backhuys, Leiden, Holanda. 198 p.

Kaas, P. \& R.A. Van Belle. 1987. Monograph of living chitons. Vol. 3, Schnochitonidae: Chaetopleurinae, Ischnochitoninae (pars). E.J. Brill / W. Backhuys, Leiden, Holanda. 302 p.

Kaas, P. \& R.A. Van Belle. 1990. Monograph of living chitons (Mollusca: Polyplacophora), Vol. 4, Suborden Ischnochitonina: Ischnochitonidae: Ischnochitoninae (continued), additions to vols. 1, 2 and 3. E.J. Brill / W. Backhuys, Leiden, Holanda. 298 p.

Kaas, P. \& R.A. Van Belle. 1994. Monograph of living chitons (Mollusca: Polyplacophora), Vol. 5, Suborden Ischnochitonina: Ischnochitonidae (concluded); Callistoplacinae, Mopalidae, additions to vols. 1-4. E.J. Brill / W. Backhuys, Leiden, Holanda. 402 p.

Kaas, P. \& R.A. Van Belle. 1998. Catalogue of living chitons (Mollusca, Polyplacophora). Backhuys, Leiden, Holanda. 204 p.

Keen, A.M. 1971. Sea shells of tropical West America: marine mollusks from Baja California to Peru. Stanford University, Stanford, California, EUA. 1064 p.

Menge, B.A. \& G.M. Branch. 2001. Rocky intertidal communities, p 221-251. In M.D. Bertness, S.D. Gaines \& M. Hay (eds). Marine Community Ecology. Sinauer Associates, Sunderland, Massachussetts, EUA.

Reyes-Gómez, A.\& M.A. Salcedo-Vargas. 2002. The recent mexican chiton (Mollusca: Polyplacophora) species. Festivus 34: 17-27.

Slieker, F.J.A. 2000. Chitons of the world: an illustrated synopsis of recent Polyplacophora. L'Informatore Piceno, Italia. 154 p.

Watters, G.T. 1990. A review of the recent eastern Pacific Acanthochitoninae (Mollusca: Polyplacophora: Cryptoplacidae) with the description of a new genus, Americhiton. Veliger 33: 241-271.

Wishart, D. 1969. An algorithm for hierarchical classifications. Biometrics 25:165-170. 